\title{
Impact of Various Sizing Metrics on Female Donor to Male Recipient Heart Transplant Outcomes
}

\author{
Nicholas Hess ${ }^{1}$, Gavin Hickey ${ }^{2}$, Ibrahim Sultan ${ }^{3}$, Yisi Wang ${ }^{2}$, and Arman Kilic ${ }^{2}$ \\ ${ }^{1}$ University of Pittsburgh Medical Center \\ ${ }^{2}$ University of Pittsburgh Medical Center Health System \\ ${ }^{3}$ University of Pittsburgh
}

April 20, 2021

\begin{abstract}
Background: This study evaluated the impact of various sizing metrics on outcomes of female donor to male recipient orthotopic heart transplantation (OHT). Methods: We queried the United Network of Organ Sharing database to analyze all isolated, primary adult OHTs from 1/12010-3/20/2020. Patients were stratified by donor-recipient sex pairing. Logistic regression was used to investigate risk-adjusted effects of current size matching criteria (weight ratio, body mass index (BMI) ratio, predicted heart mass (pHM) ratio) on one-year post-transplant mortality. Kaplan-Meier analysis was used to compare posttransplant survival among cohorts. Results: A total of 22,450 patients were analyzed, of which 3,019 (13.4\%) underwent female-to-male transplantation. Of sex-matched pairs, female-to-male donation had the lowest proportion of undersized hearts using weight and BMI ratio metrics $(10.5 \%$ and $5.2 \%)$ but had the highest proportion of undersizing using pHM metrics $(48.1 \%)($ all $\mathrm{P}<0.001)$. Female-to-male recipients had the lowest rate of unadjusted one-year survival (90.0\%, $\mathrm{P}=0.0169)$, and increased hazards of mortality after risk adjustment (OR 1.17, 95\% CI 1.01-1.36, $\mathrm{P}=0.034)$ ). Undersizing using pHM (donor-recipient ratio < 0.85) was the only metric found to be associated in increased mortality after risk adjustment (OR 1.32, 95\% CI 1.02 to $1.71, \mathrm{P}=0.035$ ). Conclusions: Female-to-male heart transplantation has the worst survival of all sex-matching combinations. Although female donors in this cohort are appropriately sized using traditional metrics, half are under-sized using pHM. This, combined with its strong association with mortality, underscores the importance of routine pHM assessment when evaluating female donors for male recipients.
\end{abstract}

\section{Introduction}

Orthotopic heart transplantation (OHT) remains the gold standard treatment for advanced stage heart failure $^{1}$. When selecting an appropriate donor for each intended recipient, it is paramount that donor-torecipient sizing is within acceptable standards in order to meet the cardiac output demands of its recipient ${ }^{2,3}$. As a donor heart under consideration typically cannot be directly inspected prior to the deployment of a harvesting team, a number of different metrics have been utilized in clinical practice as a quick tool for sizing. These metrics include height, weight, and/or body mass index (BMI) ratios, and even predicted heart mass $(\mathrm{pHM})$ calculations ${ }^{2-6}$. For weight sizing, some experts have advocated to finding a donor-recipient match pair with [?] $30 \%$ weight discrepancy ${ }^{7}$, but others advocate for a more conservative [?] $20 \%$ discrepancy $^{8,9}$. However, the correlations with some of these measurements to actual heart size have come into question.

In addition to choosing a donor heart of optimal size, considerations must also be taken into donor and recipient gender. Prior study has suggested that female to male (FtoM) donation may be associated with increased risk of posttransplant mortality ${ }^{10-13}$. A possible explanation is that a donor female graft may be more likely to be under-sized when paired with a male recipient, resulting in a cardiac supply-demand mismatch in the posttransplant period. As there is no definitive consensus with regards to how donorrecipient heart sizing should be conducted, we sought to analyze the distributions of sizing in the FtoM 
OHT population using available sizing metrics. We aimed to investigate the impacts of these metrics on posttransplant survival in this potentially higher-risk transplant cohort.

\section{Patients and Methods}

\section{Data Source}

The United Network for Organ Sharing (UNOS) database was utilized for this study, which is a prospectively collected registry of all United States solid organ transplants since 1987. For this study, we analyzed patients who underwent isolated, primary OHT from January 1, 2010 to March 20, 2020. Patient and medical center identifiers were excluded from the analysis and manuscript. This study was approved by the Institutional Review Board at the University of Pittsburgh.

\section{Study Design}

For this analysis, we included adult patients (18 years or older) who underwent OHT within the aforementioned study period. Patients who underwent concurrent simultaneous OHT with any other solid organ transplants, as well as heterotopic heart transplants were excluded. Patients were stratified based on donorrecipient sex pairing.

The primary outcome of this study was one-year posttransplant survival. Secondary outcomes included postoperative complications, hospital length of stay, and rates of drug-treated one-year acute rejection. Predictors of one-year mortality were also modeled.

\section{Statistical Analysis}

Continuous data are presented as mean ( \pm standard deviation) for normally distributed variables or median (interquartile range (IQR)) for non-normally distributed variables. Categorical data are displayed as number (percentage). Preoperative baseline characteristics were compared between groups using Student's t-test for normally distributed continuous variables and Wilcoxon rank sum test for non-normally distributed variables. Categorical variables were compared using chi square and Fisher's exact test as indicated.

Kaplan Meier survival analysis was used to compare actuarial survival between patient cohorts. Multivariable logistic regression was performed to evaluate the risk-adjusted predictors of one-year posttransplant mortality. Statistical analysis was performed using Stata version 16 (StataCorp, College Station, TX, USA).

\section{Results}

This study included a total of 22,450 OHT recipients. Of these, 13,389 (59.6\%) were male and received a heart from a male donor (MtoM), 3,660 (16.3\%) were female and received a heart from a female donor (FtoF). A total of 2,292 (10.2\%) recipients were female and received a heart from a male donor (MtoF), and 3,019 (13.4\%) were male and received a heart from a female donor (FtoM). Baseline recipient, donor, and transplant characteristics are presented in Table 1 . Median age was highest in the FtoM cohort, and this cohort had both the highest percentage of patients with ischemic cardiomyopathy and lowest percentage of patients with nonischemic cardiomyopathy (all $\mathrm{P}<0.001$ ).

Distributions of donor-to-recipient sizing are presented inTable 2 . For the weight and BMI metrics, a cutoff of $>20 \%$ donor-to-recipient discrepancy, or a donor-to-recipient ratio $<0.8$ were used to define "undersized". A $>15 \%$ donor-to-recipient discrepancy, or a donor-to-recipient ratio $<0.85$ were used to define undersizing using the pHM metric. These thresholds were based on prior publications ${ }^{6,8,9}$. FtoM recipients were least commonly undersized based on weight and BMI sizing metrics (both $\mathrm{P}<0.001$ ). When using the pHM sizing metric, FtoM recipients were most commonly undersized $(48.1 \%$ undersized, $\mathrm{P}<0.001)$.

Following OHT, the FtoM cohort experienced the highest incidence of renal failure requiring dialysis (Table 3 ) $(\mathrm{P}=0.039)$. Rates of posttransplant stroke and pacemaker placement were equivalent. Thirty-day mortality was highest in the FtoM cohort and lowest in the MtoF cohort (4.6\% vs 2.9\%, $\mathrm{P}=0.003)$. Drug-treated acute graft rejection within the first year of transplantation was highest in the FtoF cohort and lowest in the MtoM cohort $(16.5 \%$ vs $9.7 \%, \mathrm{P}<0.001)$. 
Median follow up time was 2.98 years (IQR 1.00 to 5.63 years). At one year, actuarial survival was greatest in the MtoF cohort (92.0\%). Actuarial survival was lowest in the FtoM cohort (90.0\%, $\mathrm{P}=0.0169)$. Kaplan Meier survival comparison of all donor-recipient sex pairs are displayed in Figure 1 .

A multivariable analysis was performed to identify independent risk-adjusted predictors of one-year mortality following OHT. This analysis adjusted for multiple independent predictors of mortality including race, heart failure etiology, body mass index, pretransplant mechanical ventilation or use of intra-aortic balloon pump, ventricular assist device, donor age, and graft cold ischemia time. Predictors are presented in Table 4 . In this analysis, both FtoF (HR 1.29, 95\% CI 1.14 to 1.62, $\mathrm{P}=0.001$ ) and FtoM (OR 1.17, 95\% CI 1.01 to 1.36, $\mathrm{P}=0.034)$ donation were associated with increased odds for mortality (in relation to MtoM).

Because the FtoM cohort was found to have the lowest unadjusted 30-day and one-year mortality, we investigated the impact of various donor-recipient sizing metrics on posttransplant outcomes. In a univariable analysis, donor weight undersizing was associated with a $1 \%$ increase in odds for one-year mortality for each $1 \%$ undersized in relation to the recipient, though these findings did not reach statistical significance (OR $1.01,95 \%$ CI 1.00 to $1.01, \mathrm{P}=0.055)$. This relation was also observed with undersizing based on $\mathrm{pHM}$, and did not meet statistical significance (OR 1.01, 95\% CI 1.00 to 1.02, $\mathrm{P}=0.170$ ). Undersizing by BMI was found to have a significant association with one-year mortality (per 1\% undersized, OR 1.01, 95\% CI 1.00 to 1.01, $\mathrm{P}=0.008$ ).

Multivariable logistic regression was performed to identify independent risk factors of one-year posttransplant mortality in the FtoM cohort. In this model, donor-recipient sizing metrics were adjusted for recipient age, year of transplantation, heart failure etiology, total bilirubin and serum creatinine, pretransplant mechanical ventilation, ventricular assist device, and waitlist time. Full models are displayed inSupplemental Tables $1-3$. In these models, donor heart undersizing was significantly associated increased odds of mortality for all three sizing metrics (Table $\mathbf{5}$ ). For each sizing metric, a $1 \%$ increase in the degree of undersizing was associated with a $1 \%$ increase in odds of one-year mortality. A large change in donor-recipient sizing ratio (50\% reduction, or recipient value twice that of the donor value) was associated with significant increases in odds of mortality. Of sizing metrics, a $50 \%$ reduction in $\mathrm{pHM}$ ratio had the highest odds of mortality (OR $3.74,95$ CI 1.25 to $11.16, \mathrm{P}=0.018)$.

Donor sizing metrics were also analyzed as categorical variables with weight and BMI ratios of $<0.8$ considered undersized, and with a pHM $<0.85$ considered undersized. In these analyses after risk adjustment, both undersizing based on weight (OR 1.40, 95\% CI 0.94 to 2.07, $\mathrm{P}=0.095$ ) and BMI (OR 1.47, 95\% CI 0.84 to $2.58, \mathrm{P}=0.180$ ) were not significantly associated with posttransplant mortality. Undersizing using the $\mathrm{pHM}$ metric was associated with a significant increase in mortality (OR 1.32, 95\% CI 1.02 to $1.71, \mathrm{P}=0.035$ ).

\section{Discussion}

In this analysis, we found FtoM donation to be associated with the lowest rate of 30-day and one-year survival in comparison of all sex-match pairs, and female donation (both FtoF and FtoM) to be associated with increased risk adjusted hazards for one-year mortality. There was incremental risk of mortality with incremental degree of donor heart undersizing using all three donor-to-recipient sizing metrics within the FtoM cohort. However, based on prior sizing thresholds, only pHM was found to have significant impacts on mortality. With large degrees of undersizing, pHM was found to have the strongest associations with mortality. Additionally, we found that approximately half of FtoM recipients receive a donor heart with a donor-recipient $\mathrm{pHM}$ ratio that is $<0.85$.

Existing analyses investigating the impacts of donor undersizing based on weight (donor-recipient weight ratio $<0.8$ ) have had mixed conclusions ${ }^{7,8,14}$. Jayarajan and colleagues did not find associations with use of weight-undersized donors and survival in either sex-matched or MtoF recipients. However, in FtoM recipients, undersizing using weight metrics was associated with decreased median posttransplant survival (435 days, $\mathrm{P}=0.0241$ ) and risk adjusted hazards for mortality (HR 1.201, $\mathrm{P}=0.0383)^{14}$. Bergenfeldt and colleagues found weight undersizing to be associated with increased mortality, but these findings did not apply to obese recipients ${ }^{2}$. Other studies have identified undersizing based on weight to be associated with increased 
mortality in UNOS status 1 patients $^{7}$ and patients with increased pulmonary vascular resistance ${ }^{15}$. Other groups have suggested undersizing based on weight metrics to be associated with early graft failure ${ }^{16,17}$. In our analysis, we did not find donor-recipient weight ratio $<0.8$ to be significantly associated with survival in the FtoM transplant cohort.

Other groups have advocated the use of pHM as a better means of donor-recipient heart sizing ${ }^{3,6}$. Kransdorf et al studied the use of pHM in the general OHT population ${ }^{6}$. In their analysis, undersizing based on pHM (donor-recipient ratio $<0.86$ ) was associated with increased hazards for one-year mortality (HR 1.34, 95\% CI 1.13 to $1.59, \mathrm{P}<0.001$ ). In this analysis, undersizing based on weight, BMI height, or body surface area metrics were not found to have significant impacts on posttransplant survival in the general OHT population. They concluded that a minimum donor-recipient pHM ratio of 0.86 was required to sustain optimal cardiac output $^{18}$. When analyzing the FtoM cohort, we found increased risk adjusted mortality risk in those who were undersized using pHM metrics, all while no associations were found using either weight or BMI.

Prior report has suggested increased mortality following sex-mismatched transplants, but only when the recipient is male ${ }^{10}$. This decreased survival in the FtoM cohort was observed at one year, but longer-term impacts are not as well understood. A possible explanation for increased mortality in this cohort may be that there is a higher propensity for female donors to be undersized in relation to their male recipients. Previous study has suggested that an undersized donor heart may be able to increase left ventricular mass over time to adapt to increases in systemic demand ${ }^{19}$. If true, the impacts of donor undersizing may be greatest within the first year following transplantation.

The relationship between sex-pairing and donor sizing has proven rather complex. When evaluating female donors for male recipients, our study found this sex-paired combination to have the lowest rates of undersizing when using weight or BMI metrics. Furthermore, Bergenfeldt and colleagues have found no associations with FtoM donation and inappropriate weight matched donors (donor-recipient weight ratio $<0.7)^{2}$. It is possible that this propensity for undersizing and possibility for decreased survival to be known by transplanting surgeons, and that precaution is taken to not "undersize" a female donor to a male recipient when using BMI or weight-based metrics. However, when analyzing this population with the pHM metric, nearly half of the FtoM recipients were undersized (donor-recipient pHM ratio $<0.85$ ). Reed and colleagues found that pHM discrepancies of $>10-15 \%$ to be associated with increased mortality ${ }^{3}$. Such findings may account for decreased survival in the FtoM OHT population. It is possible that differences in distributions of body fat between males and females may result in differential relationships between body weight/BMI and heart size. Therefore, weight or BMI-based sizing in sex-mismatched pairs may ultimately be inaccurate. It is possible that pHM sizing may result in more appropriate sizing calculations when evaluating sex-mismatched donor pairs.

\section{Limitations}

This study was prone to limitations due to its retrospective and non-randomized design. In choosing a potential donor graft for a recipient, it is common for surgeons to employ some form of donor-to-recipient sizing prior to organ acceptance. In this study, the analysis was conducted on patients who underwent OHT, and therefore, donor-to-recipient pairing was not randomized. As a result, the true relationship between donor heart sizing and outcomes may be underestimated.

\section{Conclusions}

In this analysis of patients undergoing primary OHT, we found FtoM recipients to have the lowest unadjusted one-year posttransplant survival, and both FtoF and FtoM donation to be associated with increased hazards of mortality after risk adjustment. When looking at proportions of undersizing in these donor-to-recipient sex matching cohorts, FtoM had the lowest rates of undersizing using weight and BMI sizing metrics. However, when looking at distributions of sizing using the pHM metric, FtoM recipients were actually most frequently undersized, accounting for nearly half of the population. In this cohort, increasing degree of undersizing is associated with increased hazards of mortality using all sizing metrics. However, in large degrees of undersizing, pHM had the largest associations with increased mortality. It appears that donor-to- 
recipient sizing using weight and BMI may be misleading in this FtoM cohort, and pHM may be the best sizing metric to use for these higher-risk patient subset.

\section{Acknowledgements}

None

\section{Disclosures}

IS receives institutional research support from Medtronic and Atricure and serves as a consultant for Medtronic Vascular. AK is on the medical advisory board for Medtronic, Inc. These affiliations do not create direct conflicts with the content of this manuscript.

\section{References}

1. Khush KK, Cherikh WS, Chambers DC, et al. The International Thoracic Organ Transplant Registry of the International Society for Heart and Lung Transplantation: Thirty-sixth adult heart transplantation report - 2019; focus theme: Donor and recipient size match. J Hear Lung Transplant . 2019;38(10):10561066. doi:10.1016/j.healun.2019.08.004

2. Bergenfeldt H, Stehlik J, Höglund P, Andersson B, Nilsson J. Donor-recipient size matching and mortality in heart transplantation: Influence of body mass index and gender. J Hear Lung Transplant . 2017;36(9):940947. doi:10.1016/j.healun.2017.02.002

3. Reed RM, Netzer G, Hunsicker L, et al. Cardiac Size and Sex-Matching in Heart Transplantation: Size Matters in Matters of Sex and the Heart.JACC Hear Fail . 2014;2(1):73-83. doi:10.1016/j.jchf.2013.09.005

4. Rudasill SE, Sanaiha Y, Mardock AL, et al. Height mismatch: An overlooked component of adult heart transplant outcomes. Clin Transplant . 2020;34(6). doi:10.1111/ctr.13863

5. Shah M, Saeed O, Shin J, et al. Predicted heart mass-based size matching among recipients with moderate pulmonary hypertension: Outcomes and sex effect. J Hear Lung Transplant . 2020;39(7):648-656. doi:10.1016/j.healun.2020.01.1339

6. Kransdorf EP, Kittleson MM, Benck LR, et al. Predicted heart mass is the optimal metric for size match in heart transplantation. J Hear Lung Transplant . 2019;38(2):156-165. doi:10.1016/j.healun.2018.09.017

7. Blackbourne LH, Tribble CG, Langenburg SE, et al. Successful use of undersized donors for orthotopic heart transplantation-with a caveat.Ann Thorac Surg . 1994;57(6):1472-1476. doi:10.1016/0003-4975(94)901031

8. Sethi GK, Lanauze P, Rosado LJ, et al. Clinical significance of weight difference between donor and recipient in heart transplantation.J Thorac Cardiovasc Surg . 1993. doi:10.1016/s0022-5223(19)34077-2

9. Patel ND, Weiss ES, Nwakanma LU, et al. Impact of donor-to-recipient weight ratio on survival after heart transplantation: analysis of the United Network for Organ Sharing Database. Circulation . 2008;118(14 Suppl). doi:10.1161/CIRCULATIONAHA.107.756866

10. Ayesta A, Urrútia G, Madrid E, Vernooij RWM, Vicent L, Martínez-Sellés M. Sex-mismatch influence on survival after heart transplantation: A systematic review and meta-analysis of observational studies. Clin Transplant . 2019. doi:10.1111/ctr.13737

11. Welp H, Spieker T, Erren M, Scheld HH, Baba HA, Stypmann J. Sex Mismatch in Heart Transplantation Is Associated With Increased Number of Severe Rejection Episodes and Shorter Long-Term Survival. Transplant Proc . 2009;41(6):2579-2584. doi:10.1016/j.transproceed.2009.06.098

12. Khush KK, Kubo JT, Desai M. Influence of donor and recipient sex mismatch on heart transplant outcomes: Analysis of the International Society for Heart and Lung Transplantation Registry. J Hear Lung Transplant . 2012;31(5):459-466. doi:10.1016/j.healun.2012.02.005 
13. Martinez-Selles M, Almenar L, Paniagua-Martin MJ, et al. Donor/recipient sex mismatch and survival after heart transplantation: only an issue in male recipients? An analysis of the Spanish Heart Transplantation Registry. Transpl Int . 2015;28(3):305-313. doi:10.1111/tri.12488

14. Jayarajan SN, Taghavi S, Komaroff E, Mangi AA. Impact of low donor to recipient weight ratios on cardiac transplantation. J Thorac Cardiovasc Surg . 2013;146(6):1538-1543. doi:10.1016/j.jtcvs.2013.06.028

15. Russo MJ, Iribarne A, Hong KN, et al. Factors associated with primary graft failure after heart transplantation. Transplantation . 2010;90(4):444-450. doi:10.1097/TP.0b013e3181e6f1eb

16. D'Alessandro C, Golmard JL, Barreda E, et al. Predictive risk factors for primary graft failure requiring temporary extra-corporeal membrane oxygenation support after cardiac transplantation in adults.Eur $J$ Cardio-thoracic Surg . 2011;40(4):962-969. doi:10.1016/j.ejcts.2011.01.064

17. Kobashigawa J, Zuckermann A, Macdonald P, et al. Report from a consensus conference on primary graft dysfunction after cardiac transplantation. J Hear Lung Transplant . 2014;33(4):327-340. doi:10.1016/j.healun.2014.02.027

18. Dewey FE, Rosenthal D, Murphy DJ, Froelicher VF, Ashley EA. Does Size Matter?-Clinical Applications of Scaling Cardiac Size and Function for Body Size. Circulation . 2008;117(17):2279-2287. doi:10.1161/CIRCULATIONAHA.107.736785

19. Mather PJ, Jeevanandam V, Eisen HJ, et al. Functional and morphologic adaptation of undersized donor hearts after heart transplantation. J Am Coll Cardiol . 1995;26(3):737-742. doi:10.1016/0735-1097(95)00216Q

Table 1. Baseline donor, recipient, and transplant characteristics of patients undergoing OHT according to donor-recipient sex-paired cohorts

\begin{tabular}{|c|c|c|c|c|}
\hline & Male to Male & Female to Female & Male to Female & $\mathbf{F}$ \\
\hline & $\mathrm{N}=13,389$ & $\mathrm{~N}=3,660$ & $\mathrm{~N}=2,292$ & $\mathrm{I}$ \\
\hline \multicolumn{5}{|l|}{ Recipient Characteristics } \\
\hline Age (years) & $57(48-64)$ & $55(43-62)$ & $53(41-61)$ & 5 \\
\hline \multicolumn{5}{|l|}{ Race } \\
\hline White & $9,061(68.0 \%)$ & $2,261(62.2 \%)$ & $1,352(59.4 \%)$ & \\
\hline Black & $2,748(20.6 \%)$ & $940(25.9 \%)$ & $674(29.6 \%)$ & \\
\hline Hispanic & $1,026(7.7 \%)$ & $298(8.2 \%)$ & $178(7.8 \%)$ & \\
\hline Asian & $400(3.0 \%)$ & $112(3.1 \%)$ & $61(2.7 \%)$ & \\
\hline Other & $84(0.6 \%)$ & $22(0.6 \%)$ & $13(0.6 \%)$ & 16 \\
\hline $\operatorname{BMI}\left(\mathrm{kg} / \mathrm{m}^{2}\right)$ & $27.99(4.60)$ & $26.49(5.29)$ & $27.28(5.34)$ & \\
\hline \multicolumn{5}{|l|}{ Heart Failure Etiology } \\
\hline Non-Ischemic & $6,609(49.4 \%)$ & $2,299(63.0 \%)$ & $1,477(64.6 \%)$ & \\
\hline Ischemic & $5,252(39.3 \%)$ & $656(18.0 \%)$ & $406(17.8 \%)$ & 1 \\
\hline Congenital & $315(2.4 \%)$ & $161(4.4 \%)$ & $108(4.7 \%)$ & 75 \\
\hline Restrictive & $418(3.1 \%)$ & $168(4.6 \%)$ & $84(3.7 \%)$ & 12 \\
\hline Valvular & $159(1.2 \%)$ & $64(1.8 \%)$ & $36(1.6 \%)$ & \\
\hline Hypertrophic & $264(2.0 \%)$ & $178(4.9 \%)$ & $96(4.2 \%)$ & \\
\hline Other & $18(0.1 \%)$ & $10(0.3 \%)$ & $4(0.2 \%)$ & \\
\hline Diabetes Mellitus & $3,954(29.6 \%)$ & $830(22.8 \%)$ & $519(22.7 \%)$ & \\
\hline Total Bilirubin (mg/dL) & $1.00(1.67)$ & $0.85(1.12)$ & $0.94(1.65)$ & \\
\hline Serum Creatinine (mg/dL) & $1.30(0.59)$ & $1.08(0.62)$ & $1.08(0.55)$ & \\
\hline Positive CMV Serology & $6,985(52.2 \%)$ & $2,308(63.1 \%)$ & $1,471(64.2 \%)$ & 1 \\
\hline Pretransplant Infection & $1,457(11.2 \%)$ & $288(8.1 \%)$ & $227(10.2 \%)$ & 22 \\
\hline Transfusion on Waitlist & $3,074(23.7 \%)$ & $646(18.2 \%)$ & $462(20.8 \%)$ & \\
\hline
\end{tabular}




\begin{tabular}{|c|c|c|c|c|}
\hline & Male to Male & Female to Female & Male to Female & $\mathbf{F}$ \\
\hline Mechanical Ventilation & $160(1.2 \%)$ & $41(1.1 \%)$ & $15(0.7 \%)$ & 5 \\
\hline Intensive Care Unit & $4,108(30.7 \%)$ & $1,064(29.1 \%)$ & $806(35.2 \%)$ & \\
\hline Intravenous Inotropes & $4,371(32.6 \%)$ & $1,472(40.2 \%)$ & $908(39.6 \%)$ & \\
\hline Intra-Aortic Balloon Pump & $1,320(9.9 \%)$ & $325(8.9 \%)$ & $235(10.3 \%)$ & \\
\hline Extracorporeal Membrane Oxygenation & $172(1.3 \%)$ & $34(0.9 \%)$ & $35(1.5 \%)$ & 3 \\
\hline \multicolumn{5}{|l|}{ Donor Characteristics } \\
\hline Donor Age (years) & $29(22-39)$ & $34(24-43)$ & $27(21-37)$ & \\
\hline \multicolumn{5}{|l|}{ Donor Race } \\
\hline White & $8,487(63.4 \%)$ & $2,506(68.5 \%)$ & $1,315(57.4 \%)$ & \\
\hline Black & $2,283(17.1 \%)$ & $522(14.3 \%)$ & $385(16.8 \%)$ & \\
\hline Asian & $2,204(16.5 \%)$ & $496(13.6 \%)$ & $512(22.3 \%)$ & 37 \\
\hline Hispanic & $209(1.6 \%)$ & $93(2.5 \%)$ & $38(1.7 \%)$ & \\
\hline Other & $206(1.5 \%)$ & $43(1.2 \%)$ & $42(1.8 \%)$ & 47 \\
\hline BMI $\left(\mathrm{kg} / \mathrm{m}^{2}\right)$ & $27.26(5.43)$ & $27.98(6.82)$ & $25.11(4.84)$ & \\
\hline \multicolumn{5}{|l|}{ Mechanism of Death } \\
\hline Trauma & $7,387(55.2 \%)$ & $1,029(28.1 \%)$ & $1,318(57.6 \%)$ & \\
\hline Cardiovascular & $2,061(15.4 \%)$ & $1,123(30.7 \%)$ & $339(14.8 \%)$ & \\
\hline Drug Overdose & $1,772(13.2 \%)$ & $616(16.8 \%)$ & $218(9.5 \%)$ & \\
\hline Other & $2,167(16.2 \%)$ & $889(24.3 \%)$ & $414(18.1 \%)$ & \\
\hline Diabetes Mellitus & $410(3.1 \%)$ & $192(5.3 \%)$ & $64(2.8 \%)$ & \\
\hline Hypertension & $4,228(31.9 \%)$ & $1,169(32.3 \%)$ & $667(29.4 \%)$ & 9 \\
\hline Serum Creatinine (mg/dL) & $1.60(1.60)$ & $1.26(1.33)$ & $1.46(1.50)$ & \\
\hline Donor LVEF $<50 \%$ & $207(1.5 \%)$ & $50(1.4 \%)$ & $33(1.4 \%)$ & \\
\hline Positive CMV Serology & $7,755(58.2 \%)$ & $2,482(68.1 \%)$ & $1,406(61.6 \%)$ & \\
\hline Hepatitis C Positive & $445(3.3 \%)$ & $140(3.8 \%)$ & $52(2.3 \%)$ & \\
\hline \multicolumn{5}{|l|}{ Transplant Characteristics } \\
\hline Days Listed & $118(31-335)$ & $75(21-232)$ & $72(19-237)$ & 77 \\
\hline Donor Distance from Recipient Hospital (nautical miles) & $86(12-275)$ & $151(22-352)$ & $92.5(12-281.5)$ & 12 \\
\hline Cold Ischemic Time (hours) & $3.1(2.4-3.8)$ & $3.2(2.5-3.9)$ & $3.1(2.3-3.8)$ & \\
\hline
\end{tabular}

BMI, body mass index

CMV, cytomegalovirus

LVEF, left ventricular ejection fraction

Table 2. Incidence of donor graft undersizing according to three available sizing metrics among various donor-recipient sex-paired cohorts

\begin{tabular}{llllll}
\hline & Male to Male & Female to Female & Male to Female & Female to Male & P-Value \\
\hline Weight Ratio & $\mathbf{N = 1 3 , 3 8 9}$ & $\mathbf{N = 3 , 6 6 0}$ & $\mathbf{N = 2 , 2 9 2}$ & $\mathbf{N = 3 , 0 1 9}$ & \\
$\begin{array}{l}\text { Normal ([?]0.8) } \\
\text { Undersized }(<0.8)\end{array}$ & $11,042(82.5 \%)$ & $3,274(89.5 \%)$ & $2,003(87.4 \%)$ & $2,702(89.5 \%)$ & $<0.001$ \\
$\begin{array}{l}\text { BMI Ratio } \\
\text { Normal ([?]0.8) }\end{array}$ & $10,955(81.8 \%)$ & $3,195(87.3 \%)$ & $1,654(72.2 \%)$ & $2,861(94.8 \%)$ & $<0.001$ \\
$\begin{array}{l}\text { Undersized (<0.8) } \\
\text { pHM Ratio }\end{array}$ & $2,434(18.2 \%)$ & $465(12.7 \%)$ & $638(27.8 \%)$ & $158(5.2 \%)$ & \\
Normal ([?]0.85) & $12,361(92.3 \%)$ & $3,465(94.7 \%)$ & $2,287(99.8 \%)$ & $1,568(51.9 \%)$ & $<0.001$
\end{tabular}




\begin{tabular}{llllll}
\hline & Male to Male & Female to Female & Male to Female & Female to Male & P-Value \\
\hline Undersized $(<0.85)$ & $1,028(7.7 \%)$ & $195(5.3 \%)$ & $5(0.2 \%)$ & $1,451(48.1 \%)$ & \\
\hline
\end{tabular}

BMI, body mass index

pHM, predicted heart mass

Table. 3. Posttransplant outcomes among various sex-matched cohorts

\begin{tabular}{llllll}
\hline & Male to Male & Female to Female & Male to Female & Female to Male & P-Va \\
\hline & $\mathbf{N = 1 3 , 3 8 9}$ & $\mathbf{N}=\mathbf{3 , 6 6 0}$ & $\mathbf{N}=\mathbf{2 , 2 9 2}$ & $\mathbf{N = 3 , 0 1 9}$ & \\
Renal Failure Requiring Dialysis & $1,509(11.5 \%)$ & $404(11.3 \%)$ & $215(9.6 \%)$ & $354(11.9 \%)$ & 0.039 \\
Stroke & $384(2.9 \%)$ & $93(2.6 \%)$ & $77(3.4 \%)$ & $81(2.7 \%)$ & 0.29 \\
Permanent Pacemaker & $388(3.0 \%)$ & $102(2.8 \%)$ & $63(2.8 \%)$ & $82(2.8 \%)$ & 0.93 \\
Hospital Length of Stay (days) & $15(11-22)$ & $16(11-24)$ & $15(11-22)$ & $15(11-23)$ & $<0.00$ \\
Drug-Treated Acute Rejection & $1,276(9.7 \%)$ & $597(16.5 \%)$ & $305(13.5 \%)$ & $300(10.1 \%)$ & $<0.00$ \\
30-Day Mortality & $433(3.3 \%)$ & $126(3.6 \%)$ & $64(2.9 \%)$ & $135(4.6 \%)$ & 0.003 \\
\hline
\end{tabular}

Table 4. Multivariable Logistic Regression for one-year mortality following OHT.

\begin{tabular}{llll}
\hline & Odds Ratio & $\mathbf{9 5 \%}$ Confidence Interval & P-Value \\
\hline Age, increasing, per year & 1.02 & $1.02,1.03$ & $<0.001$ \\
Transplant year, increasing, per year & 1.08 & $1.06,1.10$ & $<0.001$ \\
Race & & & \\
White & Ref & Ref & Ref \\
Black & 1.03 & $0.90,1.17$ & 0.684 \\
Hispanic & 1.36 & $1.14,1.62$ & 0.001 \\
Asian & 1.01 & $0.75,1.35$ & 0.961 \\
Other & 0.40 & $0.16,1.00$ & 0.051 \\
Donor-Recipient Sex Match & & & \\
MtoM & Ref & Ref & Ref \\
FtoF & 1.29 & $1.12,1.49$ & 0.001 \\
MtoF & 1.19 & $1.00,1.42$ & 0.057 \\
FtoM & 1.17 & $1.01,1.36$ & 0.034 \\
BMI, increasing, per $1 \mathrm{~kg} / \mathrm{m} 2$ & 1.03 & $1.02,1.04$ & $<0.001$ \\
Heart Failure Etiology & & & Ref \\
Non-Ischemic & Ref & Ref & 0.078 \\
Ischemic & 1.11 & $0.99,1.25$ & $<0.001$ \\
Congenital & 3.04 & $2.32,4.00$ & 0.002 \\
Restrictive & 1.50 & $1.16,1.94$ & 0.824 \\
Valvular & 0.95 & $0.59,1.52$ & 0.534 \\
Hypertrophic & 1.11 & $0.79,1.56$ & $<0.001$ \\
Other & 5.04 & $2.16,11.76$ & 0.128 \\
Diabetes mellitus & 1.09 & $0.98,1.22$ & $<0.001$ \\
Total bilirubin, increasing, per $1 \mathrm{mg} / \mathrm{dL}$ & 1.16 & $1.12,1.19$ & $<0.001$ \\
Serum creatinine, increasing, per $1 \mathrm{mg} / \mathrm{dL}$ & 1.16 & $1.08,1.24$ & $<0.001$ \\
Pretransplant mechanical ventilation & 3.10 & $2.20,4.36$ & 0.008 \\
Pretransplant ICU & 1.19 & $1.05,1.36$ & 0.008 \\
Pretransplant IABP & 1.31 & $1.07,1.59$ & \\
& & &
\end{tabular}




\begin{tabular}{llll}
\hline & Odds Ratio & 95\% Confidence Interval & P-Value \\
\hline VAD device & & & \\
None & Ref & Ref & Ref \\
LVAD & 1.34 & $1.19,1.52$ & $<0.001$ \\
RVAD & 2.24 & $0.93,5.39$ & 0.073 \\
TAH & 4.08 & $2.86,5.81$ & $<0.001$ \\
LVAD + RVAD & 1.86 & $1.35,2.56$ & $<0.001$ \\
Donor age, increasing, per year & 1.01 & $1.00,1.01$ & $<0.001$ \\
Graft cold ischemia time, increasing, per hour & 1.18 & $1.13,1.23$ & $<0.001$ \\
Waitlist time, increasing, per day & 1.00 & $1.00,1.00$ & 0.137 \\
\hline
\end{tabular}

BMI, body mass index

ICU, intensive care unit

LVAD, left ventricular assist device

FtoM, female-to-male

FtoF, female-to-female

MtoF, male-to-female

MtoM, male-to-male

RVAD, right ventricular assist device

$\mathrm{TAH}$, total artificial heart

VAD, ventricular assist device

Table 5. Multivariable logistic regression for one-year mortality of OHT recipients undergoing female-tomale donation

\begin{tabular}{llll}
\hline & Odds Ratio & 95\% Confidence Interval & P-Value \\
\hline Donor-Recipient Under-Sizing, per 1\% & & & \\
Weight & 1.01 & $1.00,1.01$ & 0.013 \\
BMI & 1.01 & $1.00,1.01$ & 0.002 \\
pHM & 1.01 & $1.00,1.02$ & 0.039 \\
Donor-Recipient Ratio, per 50\% reduction & & & \\
Weight & 2.21 & $1.29,3.78$ & 0.004 \\
BMI & 2.43 & $1.49,3.99$ & $<0.001$ \\
pHM & 3.74 & $1.25,11.16$ & 0.018 \\
\hline
\end{tabular}

BMI, body mass index

pHM, predicted heart mass

Figure Legend

Figure 1. One-year unadjusted posttransplant survival of all four sex-paired recipient cohorts following orthotopic heart transplantation 


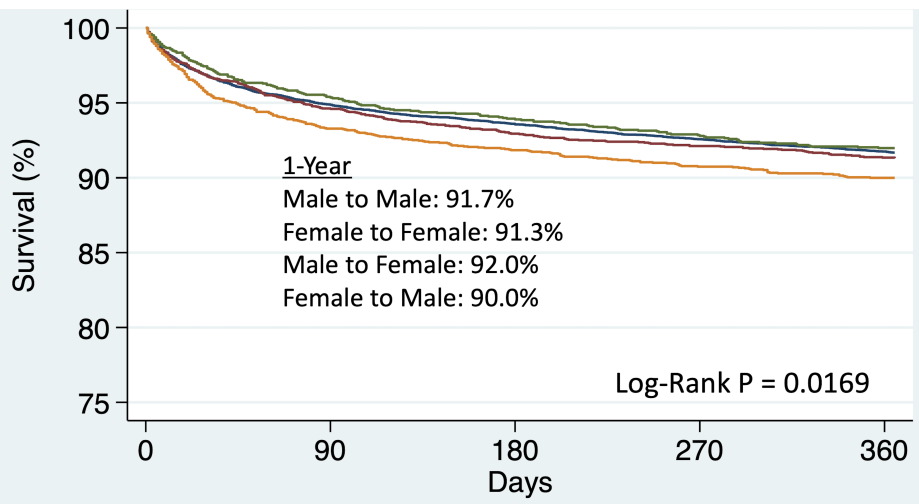

Number at risk

Male to Male1312

Female to Female 3590

Male to Female 2247

11804

Days

Female to Male 2975

3218

11300

3061

10673

10217

2034

1945

2665

2572

1840

2740

Male to Male

2448

Female to Female

Male to Female

Female to Male 